\section{Standards for helmets}

I want to thank $C M A J$ for publicizing that most domestic and imported snow sport helmets currently do not meet the Canadian Safety Association's (CSA) standards for preventing concussion and withstanding multiple impacts. ${ }^{1}$

On Mar. 19, 2007, I tabled Private Member's Bill C-412 that would prohibit the advertising, import or sale of recreational snow sport helmets that do not meet CSA's standards. Five years later, despite the advice of Health Canada and an expert panel to adopt the standards, federal health ministers refuse to do so.

My current Bill C-275 will add nonCanadian Safety Association-approved recreational snow sport helmets to the Hazardous Products Act. All that is required is the stroke of a pen by the Prime Minister.

\section{Hedy Fry MD}

Member of Parliament, Liberal Health

Critic, Liberal Party of Canada, Ottawa, Ont.

\section{Reference}

1. Sibbald B. Yes to ski helmets, but buyer beware CMAJ 2012;184:627.

CMAJ 2012. DOI:10.1503/cmaj.112-2032

\section{Emergency department visits and infections}

We read with interest the article by Quach and colleagues, ${ }^{1}$ but disagree with the authors' interpretation that the findings suggest a causal link between emergency department visits and subsequent infections. The more likely sequence of events may have been that infections in patients led to emergency department visits for nonspecific symptoms, emergency care providers did not always make the diagnosis and diagnoses were made in the subsequent week.

An essential feature of a cohort study is that patients must be free of disease at the time the exposure is assessed. In this study, ${ }^{1}$ the exposure is the emergency department visit; so, excluding infection as the cause of the emergency depart- ment visit is paramount. The authors' method of excluding infections at the time of the emergency department visit was to examine the reason for the visit. This approach is problematic because reasons for visits often match poorly with clinical diagnoses. ${ }^{2}$ For example, a complaint of "mobility impairment" from a patient from a long-term care facility does not exclude infection as an etiology. That the emergency department visit came before the diagnosis of infection in no way establishes the direction of causality - this was described by Bradford Hill in 1965 as a problem of temporality. ${ }^{3}$

An emergency department is an important site of care for residents of nursing homes, and research that suggests emergency departments may be harmful may do a disservice to patients who need such care. ${ }^{4}$ A person who got sick after visiting an emergency department may have had an illness that predated the visit. We do not dispute that any public place, including hospital emergency departments, may lead to transmission of infection, but in our opinion the evidence presented by Quach and colleagues and the methods used to gather that evidence are insufficient to support their conclusion.

\section{Timothy F. Platts-Mills MD}

School of Medicine

Philip D. Sloane MD MPH

Department of Family Medicine

University of North Carolina, Chapel Hill, NC

\section{References}

1. Quach C, McArthur M, McGeer A, et al. Risk of infection following a visit to the emergency department: a cohort study. CMAJ 2012;184:E232-9.

2. Fleischauer AT, Silk BJ, Schumacher M, et al. The validity of chief complaint and discharge diagnosis in emergency department-based syndromic surveillance. Acad Emerg Med 2004;11:1262-7.

3. Bradford Hill A. The environment and disease: association or causation? Proceedings of the Royan Society of Medicine 1965;58:295-300.

4. Wang HE, Shah MN, Allman RM, et al. Emergency department visits by nursing home residents in the United States. J Am Geriatr Soc 2011;59: 1864-72.

\section{CMAJ 2012. DOI:10.1503/cmaj.112-2034}

\section{The authors respond}

We thank Dr. Platts-Mills and Dr. Sloane for their comments ${ }^{1}$ - and we agree, as stated in the conclusion of our article, that "confirmation of these results with studies of specific types of infection with laboratory testing is required." ${ }^{2}$ We included only residents of long-term care facilities who received an emergency department discharge diagnosis other than a respiratory or a gastrointestinal infection. We screened charts of emergency departments and long-term care facilities to exclude patients with symptoms of these illnesses before or during their emergency department visit.

Although many think that residents of long-term care facilities may be more likely to have atypical presentations of infections, Berman and colleagues ${ }^{3}$ showed that "in most elderly patients who develop infection, there remain clear clinical pointers to the diagnosis. The symptoms may be absent or unreliable but the physical signs remain." In our study population, the onset of symptoms started on average 4.1 days (median 3.5) after residents returned from the emergency department, which is the typical incubation period for respiratory and gastrointestinal infections following an exposure. ${ }^{4}$

Moreover, if a resident exposed to the emergency department were incubating a respiratory or gastrointestinal infection at the time of exposure, we would have expected that transmission to also have occurred at the resident's unit in the long-term care facility. Two randomly chosen residents were matched by the unit or ward in the same long-term care facility and on the index date (return date of one resident from the emergency department). If the returning resident had been exposed in the long-term care facility before presenting to the emergency department, the resident who did not visit the emergency department would have the same chance of exposure, thus decreasing the strength of the association found.

The finding of our study is biologically plausible and is in keeping with the increased risk described by Troko and colleagues. ${ }^{5}$ They reported that use of a bus or tram within five days of symptom onset was associated with an almost sixfold increased risk of con- 